\title{
Role of Immunotherapy in the Treatment of Squamous Cell Carcinoma of the Anal Canal
}

Van K. Morris, MD, MS, and Cathy Eng, MD

\section{Abstract}

Anal cancer is a rare malignancy for which cisplatin with 5-fluorouracil is the recommended treatment for patients with metastatic disease. Because most cases of anal cancer are linked to prior infection with oncogenic strands of the human papillomavirus, immunotherapeutic approaches have been of great interest in the development of new treatments for this virally driven tumor. This article reviews the early successes of anti-PD-1 therapies and adoptive T-cell therapies for metastatic anal cancer as a potential foundation for novel combination immunotherapy trials in the future.

J Natl Compr Canc Netw 2018;16(7):903-908 doi: 10.6004/jnccn.2018.7040

Squamous cell carcinoma of the anal canal (SCCA) is an uncommon malignancy, for which more than 8,000 new diagnoses and 1,000 deaths in the United States are expected in 2018. ${ }^{1}$ Oncogenic subtypes of human papillomaviruses (HPV), including HPV-16, -18, -31, $-33,-45,-52$, and -58 , are attributed to $>90 \%$ of SCCA cases. $^{2-6}$ In 2015, the FDA approved a 9-valent vaccine ${ }^{7}$ for primary prevention of HPV-associated malignancies such as SCCA. Current CDC guidelines recommend administration of 2 doses of the HPV vaccine, at least 6 months apart, in all preteen adolescents starting at age 11 or 12 years, until the age of 22 (men) or 27 (women) years. ${ }^{8}$ For teenage or young men who have sex with other men or people with weakened immunity, the HPV vaccine is recommended until the age of 27 years. ${ }^{8}$ However, the benefits of vaccination in reducing some HPV-associated cancers may not be expected for decades, ${ }^{9}$ and the annual incidence of this cancer continues to increase. ${ }^{10}$

Patients with nonmetastatic SCCA are most commonly treated with concurrent chemoradiation with combination chemotherapy — a standard treatment paradigm

The University of Texas MD Anderson Cancer Center, Houston, Texas.

Submitted January 29, 2018; accepted for publication April 30, 2018.

The authors have disclosed that they have no financial interests, arrangements, affiliations, or commercial interests with the unchanged for the past 4 decades. ${ }^{11-15}$ However, treatment options for metastatic SCCA remain limited. The 2018 NCCN Clinical Practice Guidelines in Oncology for Anal Carcinoma recommend cisplatin/5-fluorouracil (5-FU), carboplatin/paclitaxel, or 5-FU/oxaliplatin as frontline treatment for metastatic SCCA. ${ }^{16}$ InterAACT/ EA2133 (ClinicalTrials.gov identifier: NCT02560298) is the first randomized phase II prospective study to compare 2 chemotherapy doublets, 5-FU/cisplatin versus carboplatin/paclitaxel, in patients with treatment-naïve metastatic anal cancer; results are expected to be reported in 2018. Because there are few options for this population, new, effective treatment strategies are warranted. This review highlights the role of immune-based therapies for patients with this HPV-associated malignancy.

\section{Anti-PD-1 Therapies}

In a physiologic state, immune checkpoint regulators such as PD-1 and CTLA-4 bind their respective receptors on $\mathrm{T}$ cells to abrogate T-cell activity. ${ }^{17,18}$ Because of their ability to present mutated proteins identifiable as manufacturers of any products discussed in this article or their competitors.

Correspondence: Van K. Morris, MD, MS, The University of Texas MD Anderson Cancer Center, 1515 Holcombe Boulevard, Unit 0426, Houston, TX 77030-4006. vkmorris@mdanderson.org 
Morris and Eng

"non-self" epitopes to circulating immune cells, tumor cells can be recognized thereafter for attack. To evade such antitumor activity, tumor cells also express immune checkpoint surface receptors such as PD-L1 and CTLA-4. ${ }^{19,20}$ Monoclonal antibodies interfering with these interactions therefore promote antitumor cytotoxic immune responses by disrupting this negative feedback interaction. ${ }^{20,21}$ Nivolumab and pembrolizumab are 2 such antibodies targeting PD-1 that have demonstrated durable clinical responses in various solid tumors, ${ }^{22-25}$ including viral-associated cancers, HPV and non-HPV alike. ${ }^{26,27}$ Two studies thus far have investigated the role of anti-PD-1 therapies specifically for patients with unresectable/metastatic SCCA. 28,29

The NCI9673 study was a multicenter phase II trial that formally investigated the efficacy of nivolumab in patients with unresectable or metastatic SCCA who experienced disease progression through prior chemotherapy. ${ }^{28}$ Here, 37 patients received at least one dose of nivolumab, $3 \mathrm{mg} / \mathrm{kg} \mathrm{ev}$ ery 2 weeks. After a median follow-up lasting 10.1 months, radiographic responses according to RECIST 1.1 criteria were noted in 9 patients $(2 \mathrm{com}$ plete responses [CRs], 7 partial responses [PRs]) for an objective response rate (RR) of $24 \%(95 \% \mathrm{CI}$, $15 \%-33 \%)$. Stable disease was noted in an additional 17 patients, for a disease control rate of $72 \%$ with nivolumab monotherapy in patients with metastatic SCCA. For patients experiencing response, a median $70 \%$ reduction in tumor volume from baseline measurements was seen before treatment initiation; median progression-free survival (PFS) was 4.1 months (95\% CI, 3.0-7.9 months) and median overall survival was 11.5 months (95\% CI, 7.1 monthsnot reached). Nivolumab was generally well tolerated, with grade 3 adverse events (AEs) noted in only 5 patients; no grade 4 AEs were noted. These data suggest that clinical outcomes may be improved by anti-PD-1 therapies, relative to historical response rates from smaller cases series detailing responses to second-line cytotoxic chemotherapeutic options in this population. ${ }^{30}$ This study was the first phase II study to demonstrate efficacy of immunotherapy in patients with metastatic SCCA.

Similar findings to the NCI9673 trial were noted in KEYNOTE-028, ${ }^{29}$ a phase Ib study in which the primary objectives were to describe the safety and response rate of pembrolizumab in 20 separate cohorts of patients with advanced solid malignancies that were PD-L1-positive (defined as tumors having $>1 \%$ of cells staining positive for the 22C3 antibody). Among the 24 patients with metastatic SCCA treated with pembrolizumab, PRs were detected in 4 (RR, $17 \%$; $95 \%$ CI, 5\%-37\%), with a total disease control rate of $58 \%$. Median PFS (3.0 months) and median overall survival (9.3 months) with pembrolizumab were similar to those reported with nivolumab. Grade 3 treatment-related AEs were reported in 4 patients (17\%). Collectively, these 2 studies demonstrate that anti-PD-1 monotherapy can benefit a fraction of patients with unresectable or metastatic SCCA that has progressed on prior chemotherapy.

\section{Biomarkers for Response to Anti-PD-1 Therapies}

Favorable responses to immune checkpoint blockade agents in other advanced solid tumors such as melanoma, non-small cell lung cancer, and microsatellite instability-high colorectal cancer are often correlated with the presence of high somatic mutation frequencies. ${ }^{31-33}$ For patients with metastatic SCCA, whole-exome sequencing has demonstrated lower tumor mutation burdens (2.5 somatic mutations per megabase [Mb] DNA), ${ }^{34}$ similar to other results reported in a separate series of patients with nonmetastatic SCCA (3.5 genomic alterations per tumor). ${ }^{35}$ This low mutation frequency for SCCA is consistent with other HPV-associated malignancies, such as cervical cancer (2.8 mutations per Mb DNA) and HPV-positive head and neck cancer (3.4 mutations per Mb DNA). ${ }^{34}$ Given the durable responses associated with anti-PD-1 therapies across these HPV-related cancers, there must be other identifiable biomarkers for patients who derive benefit from these agents.

Analysis of limited paired tissue biopsies from patients with metastatic SCCA treated with nivolumab on the NCI9673 trial compared biomarker profiles between responders and nonresponders. ${ }^{28}$ All tumors tested were found to have detectable HPV, thereby limiting the ability to associate benefit of immunotherapy with HPV-positive status for this cohort of patients. Nonetheless, responding patients had tumors featuring not only an increased presence of activated cytotoxic $T$ cells but also a higher expression of PD-1 (on immune cells) and PD-L1 (on tumor 
cells) at baseline. Collectively, these findings suggest that SCCA tumors characterized by activated $\mathrm{T}$ cells in the tumor microenvironment with intact feedback axes that downregulate an antitumor immune response may be most susceptible to treatment benefit with immune checkpoint blockade agents.

\section{Cellular-Based Immune Therapies for Anal Cancer}

Certainly, alternative approaches capitalizing on non-immune checkpoint-based therapies hold promise for the management of SCCA across all disease stages. Adoptive T-cell therapy is a process through which autologous tumor-specific $T$ cells from a given patient are isolated, expanded ex vivo, and reinfused into the patient after a nonmyeloablative conditioning regimen and costimulation by IL-2. ${ }^{36}$ Although use of such tumor-infiltrating lymphocytes (TILs) thus far has demonstrated greatest success in lymphomas and melanoma, ${ }^{37,38}$ early results in other HPV-associated cancers generate optimism for this technology with SCCA. One series of 9 women with metastatic or locally advanced, platinum-refractory, HPV-positive cervical cancer (HPV-16: 2 patients, HPV-18: 9 patients) were treated with autologous HPV TILs. ${ }^{39} \mathrm{~T}$ cells were selected for expansion according to immunoreactivity to HPV oncoproteins E6 and E7 based on the identified HPV subtype (ie, HPV-16 or -18). Clinical responses were seen in 3 patients (1 PR and 2 CRs). As of April 2017, the latter 2 patients had maintained their CRs after a follow-up of 46 and 54 months, with no evidence of disease recurrence (and while off any additional antineoplastic therapy). Myelosuppression and infection were the most frequent AEs. Demonstrating safety and efficacy of HPV TILs in patients with otherwise incurable cervical cancer has provided rationale for applying this therapy to other HPV-associated malignancies, such as SCCA.

Investigators further characterized the tumor features for these 2 patients with CRs to this adoptive TIL therapy..$^{40}$ Both patients had TILs that demonstrated immune recognition to HPV oncoproteins E6 or E7, but not to any of the other HPV-produced viral proteins. Interestingly, TILs from both patients were also reactive against somatically (nonviral) mutated proteins in genes like SETDB1, METTL17, and $A L D H 1 A 1$, and, in one of these patients, to a cancer germline antigen (KK-LC-1). T-cell receptor sequencing determined that the TILs recognizing the somatic mutations as immune neoantigens were present at higher frequencies relative to TILs recognizing HPV viral antigens. Flow cytometry studies revealed that these TIL populations (reactive to HPV proteins and somatic mutations alike) were more common in the PD-1-positive T-cell subgroups, relative to PD-1-negative T-cell populations). In addition, these TILs persisted at higher levels not only during tumor regression but also during remission, likely driving the sustained complete clinical responses for these 2 patients. Collectively, these findings, although in a small subset, provide evidence that immunogenicity of antitumor lymphocytes for HPV-associated malignancies may be driven most dominantly toward selection of nonviral, somatically mutated antigens. Whether such a profound activity against these tumors by adoptive TIL therapy can be strengthened through use of concomitant anti-PD-1 blockade in SCCA remains unclear currently, but is a very exciting prospect.

Another study has reported outcomes with HPV-positive cancers treated with T cells modified to react against the HLA-A 02:01-specific E6 antigen. ${ }^{41}$ In this study, 4 patients had metastatic anal cancer, 2 of whom had PRs to therapy (lasting 3 and 6 months). The patient with a PR lasting 6 months demonstrated a sizable reduction of tumor volume that permitted metastatic resection of remnant disease. Use of T cells engineered to recognize intracellular viral proteins holds promise based on these early results suggesting antitumor activity, and warrants further study in patients with metastatic anal cancer.

\section{Non-T-Cell-Based Immunotherapies for SCCA}

Alternative immune-related therapies other than checkpoint blockade antagonists and autologous $\mathrm{T}$ cells have also been tested in early-phase clinical trials for patients with locoregional and metastatic SCCA. For example, ADXS11-001 is a bioengineered, nonvirulent Listeria monocytogenes vector designed to generate an antitumor response against HPV-associated malignancies. ${ }^{42}$ These Listeria are genetically modified to produce fusion peptides that excrete HPV oncoprotein E7 into the bacterial cytoplasm. Phagocytosis of Listeria by dendritic cells 
Morris and Eng

results in activation of an immune response against the bacteria, and eventually in lysis of cytoplasmic content, including the E7 fusion peptide, for further processing, activation of helper $T$ cells, and antibody production. Ultimately, degraded E7 oncoprotein fragments are loaded onto major histocompatibility complex grooves for recognition, recruitment, and activation of E7-specific T cells. Cytotoxic T cells are then able to mount an immune attack against tumor cells that harbor and present HPV-specific proteins.

ADXS11-001 first demonstrated encouraging results in a cohort of patients with squamous cell carcinoma or adenocarcinoma of the cervix. ${ }^{43}$ In the initial portion of this Simon 2-stage, phase II study, 26 patients with incurable disease were treated with ADXS11-001; 1 patient experienced a partial radiographic response and 9 patients had stable disease. Most patients (56\%) who received all planned doses per protocol remained alive after 12 months on study. All patients experienced treatment-related AEs, most of which were infusion-related reactions, including fevers, chills, and fatigue. Given that these patients had been heavily pretreated for their incurable disease, these results provided initial hope for disease control in HPV-associated malignancies.

Based on the promising strategy in the cervical cancer setting, a separate single-arm phase II study of ADXS11-001 monotherapy was completed in 29 patients with metastatic SCCA. ${ }^{44}$ One patient had a confirmed partial radiographic response lasting $>6$ months who, incidentally, had previously benefitted from nivolumab before treatment on this study. An additional 7 patients had stable disease, for a disease control rate of $28 \%$. More than 20\% remained without evidence of disease progression after 6 months on treatment. AEs were similar in nature and frequency to those reported in the aforementioned study of ADXS11-001 in cervical cancer. ${ }^{43}$ Again, these preliminary findings provide further support for the use of novel immunotherapeutic approaches in patients with metastatic SCCA.

ADXS11-001 has also been tested in patients with nonmetastatic SCCA receiving concurrent chemoradiation with 5 -FU and mitomycin C. ${ }^{45}$ Among 10 patients treated, $8(80 \%)$ demonstrated a complete clinical response by 6 months on study. There was 1 grade 5 AE (cardiopulmonary) unrelated to ADXS11001 treatment. After almost 3 years of follow-up, 8 of
9 patients (89\%) remained without evidence of recurrence. Although the benefit of ADXS11-001 addition remains unclear in a setting in which cure rates are similarly high with chemoradiation alone, this trial emphasized the need for extending the benefit of novel immunotherapy approaches as a means to improve long-term outcomes in patients with locoregional and nonmetastatic SCCA.

\section{Future Directions for Immunotherapy in SCCA}

The efficacy of immune monotherapies for metastatic SCCA not only offers immediate potential new options for afflicted patients, but also warrants further evaluation in the context of combination approaches to deepen an antitumor immune response. For example, the addition of ipilimumab to nivolumab has demonstrated durable responses in approximately $40 \%$ patients with metastatic melanoma and non-small cell lung cancer. ${ }^{46,47}$ The addition of anti-CTLA-4 therapy to an anti-PD-1 agent is promising and of great interest in metastatic SCCA. An upcoming amendment to the NCI9673 study randomizing patients in a 1:1 fashion to nivolumab with or without ipilimumab in metastatic SCCA was expected to begin enrolling patients in late 2018 across the NCI Experimental Therapeutics Clinical Trials Network. Similarly, based on the translational results previously described in the NCI9673 study showing higher coexpression of TIM-3 and LAG-3 in PD-1-positive SCCA tumors responding to nivolumab, ${ }^{28}$ the addition of agents targeting these immune markers to further stimulate an immune-mediated tumor attack is a very reasonable approach for future trials.

Addition of anti-VEGF therapies, such as bevacizumab, to anti-PD-1 agents is also of great interest for patients with metastatic SCCA. Transcriptomic comparison between patients with metastatic melanoma who did or did not respond to pembrolizumab showed that the latter had higher gene expression patterns for angiogenesis and tissue hypoxia, ${ }^{48}$ supporting the notion of increased VEGF signaling as a mediator for de novo resistance to immune checkpoint blockade therapies in solid tumors. Based on these data, a phase II pilot trial of the anti-PD-L1 antibody atezolizumab in combination with bevacizumab was recently completed in patients with met- 
astatic HPV-associated malignancies, most of whom had SCCA. These greatly anticipated results are expected to be reported in late 2018 .

Although recent successes with immunotherapy have generated promising early results in patients with metastatic SCCA, most patients in these trials have not shown durable responses. Through collaborations in the International Rare Cancers Initiative, correlative studies performed for future research are expected to inform more on additional mechanisms of resistance to immune checkpoint blockade therapy in patients with metastatic SCCA. Novel immune-based approaches building on translational work with next-generation immunotherapy trials are warranted, with the goal of broadening the clinical benefit of immunotherapy in this population. Given the common viral driver in pathogenesis across HPV-associated malignancies, it is expected that any advances in the treatment of anal cancer could be applied to benefit patients with not only this disease but also head and neck, cervical, vaginal/vulvar, and penile cancers.

\section{References}

1. Siegel RL, Miller KD, Jemal A. Cancer Statistics, 2017. CA Cancer J Clin 2017;67:7-30.

2. De Vuyst H, Clifford GM, Nascimento MC, et al. Prevalence and type distribution of human papillomavirus in carcinoma and intraepithelial neoplasia of the vulva, vagina and anus: a meta-analysis. Int J Cancer 2009;124:1626-1636.

3. Frisch M, Glimelius B, van den Brule AJ, et al. Sexually transmitted infection as a cause of anal cancer. N Engl J Med 1997;337:1350-1358.

4. Hoots BE, Palefsky JM, Pimenta JM, Smith JS. Human papillomavirus type distribution in anal cancer and anal intraepithelial lesions. Int J Cancer 2009; 124:2375-2383.

5. Morris VK, Rashid A, Rodriguez-Bigas M, et al. Clinicopathologic features associated with human papillomavirus/p16 in patients with metastatic squamous cell carcinoma of the anal canal. Oncologist 2015;20:12471252.

6. Serup-Hansen E, Linnemann D, Skovrider-Ruminski W, et al. Human papillomavirus genotyping and p16 expression as prognostic factors for patients with American Joint Committee on Cancer stages I to III carcinoma of the anal canal. J Clin Oncol 2014;32:1812-1817.

7. Joura E, Bautista O, Luxembourg A. A 9-valent HPV vaccine in women. $\mathrm{N}$ Engl J Med 2015;372:2568-2569.

8. Human Papillomavirus (HPV) Vaccination: What Everyone Should Know. Centers for Disease Control and Prevention web site. Available at: https://www.cdc.gov/vaccines/vpd/hpv/public/index.html. Accessed January 27, 2018.

9. Pytynia KB, Dahlstrom KR, Sturgis EM. Epidemiology of HPV-associated oropharyngeal cancer. Oral Oncol 2014;50:380-386.

10. Johnson LG, Madeleine MM, Newcomer LM, et al. Anal cancer incidence and survival: the Surveillance, Epidemiology, and End Results experience, 1973-2000. Cancer 2004;101:281-288.

11. Nigro ND, Vaitkevicius VK, Considine B Jr. Combined therapy for cancer of the anal canal: a preliminary report. Dis Colon Rectum 1974;17:354356.

12. Northover J, Glynne-Jones R, Sebag-Montefiore D, et al. Chemoradiation for the treatment of epidermoid anal cancer: 13-year follow-up of the first randomised UKCCCR Anal Cancer Trial (ACT I). Br J Cancer 2010;102:1123-1128.

13. Flam M, John M, Pajak TF, et al. Role of mitomycin in combination with fluorouracil and radiotherapy, and of salvage chemoradiation in the definitive nonsurgical treatment of epidermoid carcinoma of the anal canal: results of a phase III randomized intergroup study. J Clin Oncol $1996 ; 14: 2527-2539$

14. James RD, Glynne-Jones R, Meadows HM, et al. Mitomycin or cisplatin chemoradiation with or without maintenance chemotherapy for treatment of squamous-cell carcinoma of the anus (ACT II): a randomised, phase 3 , open-label, 2 × 2 factorial trial. Lancet Oncol 2013;14:516-524.

15. Gunderson LL, Winter KA, Ajani JA, et al. Long-term update of US GI intergroup RTOG 98-11 phase III trial for anal carcinoma: survival, relapse, and colostomy failure with concurrent chemoradiation involving fluorouracil/mitomycin versus fluorouracil/cisplatin. J Clin Oncol 2012;30:4344-4351.

16. Benson AB III, Arnoletti JP, Bekaii-Saab T, et al. NCCN Clinical Practice Guideline in Oncology: Anal Carcinoma. Version 2.2012. To view the most recent version of these guidelines, visit NCCN.org. Accessed June $1,2018$.

17. Fife BT, Bluestone JA. Control of peripheral T-cell tolerance and autoimmunity via the CTLA-4 and PD-1 pathways. Immunol Rev 2008;224:166-182.

18. Krummel MF, Allison JP. CD28 and CTLA-4 have opposing effects on the response of T cells to stimulation. J Exp Med 1995;182:459-465.

19. Ishida Y, Agata Y, Shibahara K, Honjo T. Induced expression of PD-1, a novel member of the immunoglobulin gene superfamily, upon programmed cell death. EMBO J 1992;11:3887-3895.

20. Leach DR, Krummel MF, Allison JP. Enhancement of antitumor immunity by CTLA-4 blockade. Science 1996;271:1734-1736.

21. Hirano F, Kaneko K, Tamura H, et al. Blockade of B7-H1 and PD-1 by monoclonal antibodies potentiates cancer therapeutic immunity. Cancer Res 2005;65:1089-1096.

22. Robert C, Long GV, Brady B, et al. Nivolumab in previously untreated melanoma without BRAF mutation. N Engl J Med 2015;372:320-330.

23. Motzer RJ, Escudier B, McDermott DF, et al. Nivolumab versus everolimus in advanced renal-cell carcinoma. N Engl J Med 2015;373:1803-1813.

24. Garon EB, Rizvi NA, Hui R, et al. Pembrolizumab for the treatment of non-small-cell lung cancer. N Engl J Med 2015;372:2018-2028.

25. Bellmunt J, de Wit R, Vaughn DJ, et al. Pembrolizumab as second-line therapy for advanced urothelial carcinoma. N Engl J Med 2017;376:1015_ 1026.

26. Ferris RL, Blumenschein G Jr, Fayette J, et al. Nivolumab for recurrent squamous-cell carcinoma of the head and neck. $N$ Engl J Med 2016;375:1856-1867.

27. Nghiem PT, Bhatia S, Lipson EJ, et al. PD-1 Blockade with pembrolizumab in advanced Merkel-cell carcinoma. N Engl J Med 2016;374:2542-2552.

28. Morris VK, Salem ME, Nimeiri H, et al. Nivolumab for previously treated unresectable metastatic anal cancer (NCI9673): a multicentre, single-arm, phase 2 study. Lancet Oncol 2017;18:446-453.

29. Ott PA, Piha-Paul SA, Munster P, et al. Safety and antitumor activity of the anti-PD-1 antibody pembrolizumab in patients with recurrent carcinoma of the anal canal. Ann Oncol 2017;28:1036-1041.

30. Eng C, Chang GJ, You YN, et al. The role of systemic chemotherapy and multidisciplinary management in improving the overall survival of patients with metastatic squamous cell carcinoma of the anal canal. Oncotarget 2014;5:11133-11142.

31. Cancer Genome Atlas Network. Comprehensive molecular characterization of human colon and rectal cancer. Nature 2012;487:330-337.

32. Le DT, Uram JN, Wang H, et al. PD-1 blockade in tumors with mismatchrepair deficiency. N Engl J Med 2015;372:2509-2520.

33. Alexandrov LB, Nik-Zainal S, Wedge DC, et al. Signatures of mutational processes in human cancer. Nature 2013;500:415-421.

34. Morris V, Rao X, Pickering C, et al. Comprehensive genomic profiling of metastatic squamous cell carcinoma of the anal canal. Mol Cancer Res 2017;15:1542-1550.

35. Chung JH, Sanford E, Johnson A, et al. Comprehensive genomic profiling of anal squamous cell carcinoma reveals distinct genomically defined classes. Ann Oncol 2016;27:1336-1341. 
Morris and Eng

36. Hinrichs CS, Rosenberg SA. Exploiting the curative potential of adoptive T-cell therapy for cancer. Immunol Rev 2014;257:56-71.

37. Rosenberg SA, Yang JC, Sherry RM, et al. Durable complete responses in heavily pretreated patients with metastatic melanoma using T-cell transfer immunotherapy. Clin Cancer Res 2011;17:4550-4557.

38. Kochenderfer JN, Dudley ME, Feldman SA, et al. B-cell depletion and remissions of malignancy along with cytokine-associated toxicity in a clinical trial of anti-CD19 chimeric-antigen-receptor-transduced T cells. Blood 2012;119:2709-2720.

39. Stevanovic S, Draper LM, Langhan MM, et al. Complete regression of metastatic cervical cancer after treatment with human papillomavirustargeted tumor-infiltrating T cells. J Clin Oncol 2015;33:1543-1550.

40. Stevanovic S, Pasetto A, Helman SR, et al. Landscape of immunogenic tumor antigens in successful immunotherapy of virally induced epithelial cancer. Science 2017;356:200-205.

41. Hinrichs CS, Doran SL, Stevanovic S, et al. A phase I/II clinical trial of E6 T-cell receptor gene therapy for human papillomavirus (HPV)-associated epithelial cancers [abstract]. J Clin Oncol 2017;35(Suppl):Abstract 3009.

42. Miles BA, Monk BJ, Safran HP. Mechanistic insights into ADXS11-001 human papillomavirus-associated cancer immunotherapy. Gynecol Oncol Res Pract 2017;4:9.
43. Huh WK, Dizon DS, Powell MA, et al. ADXS11-001 immunotherapy in squamous or non-squamous persistent/recurrent metastatic cervical cancer: results from stage I of the phase II GOG/NRG0265 study [abstract]. J Clin Oncol 2016;34(Suppl):Abstract 5516.

44. Eng C, Fakih M, Armin M, et al. P2 study of ADXS11-001 immunotherapy in patients with persistent/recurrent, surgically unresectable locoregional, or metastatic squamous cell anal cancer [abstract]. Ann Oncol 2017;28(Suppl 5):Abstract 537P.

45. Safran H, Leonard KL, DiPetrillo TA, et al. ADXS11-001 Lm-LLO immunotherapy, mitomycin, 5-fluorouracil (5-FU) and intensitymodulated radiation therapy (IMRT) for anal cancer [abstract]. J Clin Oncol 2017;35(Suppl):Abstract e15072.

46. Wolchok JD, Chiarion-Sileni V, Gonzalez R, et al. Overall survival with combined nivolumab and ipilimumab in advanced melanoma. N Engl Med 2017;377:1345-1356.

47. Hellmann MD, Rizvi NA, Goldman JW, et al. Nivolumab plus ipilimumab as first-line treatment for advanced non-small-cell lung cancer (CheckMate 012): results of an open-label, phase 1, multicohort study. Lancet Oncol 2017;18:31-41.

48. Hugo W, Zaretsky JM, Sun L, et al. Genomic and transcriptomic features of response to anti-PD-1 therapy in metastatic melanoma. Cell 2016;165:3544.

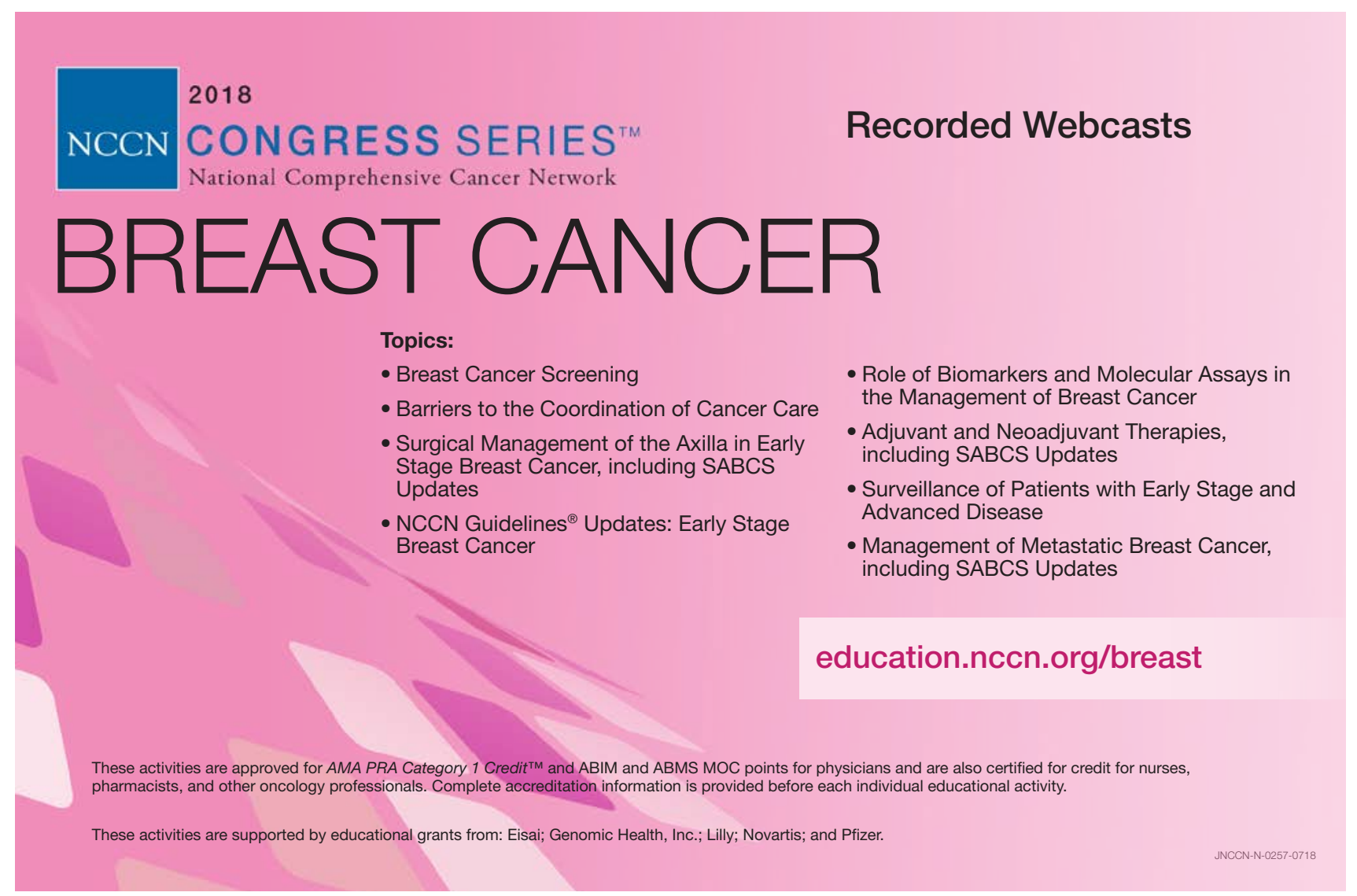

